\title{
A New Method for Defuzzification and Ranking of Fuzzy Numbers Based on the Statistical Beta Distribution
}

\author{
A. Rahmani, F. Hosseinzadeh Lotfi, M. Rostamy-Malkhalifeh, and T. Allahviranloo \\ Department of Mathematics, Science and Research Branch, Islamic Azad University, Tehran, Iran
}

Correspondence should be addressed to M. Rostamy-Malkhalifeh; mohsen_rostamy@yahoo.com

Received 25 March 2016; Revised 22 June 2016; Accepted 18 October 2016

Academic Editor: Rustom M. Mamlook

Copyright (C) 2016 A. Rahmani et al. This is an open access article distributed under the Creative Commons Attribution License, which permits unrestricted use, distribution, and reproduction in any medium, provided the original work is properly cited.

Granular computing is an emerging computing theory and paradigm that deals with the processing of information granules, which are defined as a number of information entities grouped together due to their similarity, physical adjacency, or indistinguishability. In most aspects of human reasoning, these granules have an uncertain formation, so the concept of granularity of fuzzy information could be of special interest for the applications where fuzzy sets must be converted to crisp sets to avoid uncertainty. This paper proposes a novel method of defuzzification based on the mean value of statistical Beta distribution and an algorithm for ranking fuzzy numbers based on the crisp number ranking system on R. The proposed method is quite easy to use, but the main reason for following this approach is the equality of left spread, right spread, and mode of Beta distribution with their corresponding values in fuzzy numbers within $(0,1)$ interval, in addition to the fact that the resulting method can satisfy all reasonable properties of fuzzy quantity ordering defined by Wang et al. The algorithm is illustrated through several numerical examples and it is then compared with some of the other methods provided by literature.

\section{Introduction}

Granular computing is an emerging computing paradigm that is concerned with the processing of information entities created from the process of data abstraction and is intrinsically linked with the adjustable nature of human perception $[1,2]$. Information granularity and granular computing have been widely used in development of verbal and linguistic concepts and particularly those concerned with fuzzy and rough sets [3] and are valuable assets for creating realistic models for real-world decision-making processes, as they provide the means to understand and solve abstract problems of the real world with simplicity, clarity, good approximation, and tolerance of uncertainty $[1,4]$. Granular computing is the science of building heterogeneous and multilevel models for the processing of granular information by incorporating distinct concepts such as probabilistic sets, rough sets, and especially fuzzy sets and their membership functions into a single framework, thereby allowing the verbal, linguistic, and human-centered concepts to be processed.

Since the introduction of the term "granular computing," its related concepts have appeared in many different fields such as artificial intelligence, decision-making, and cluster analysis [5-7]. Although there have been some works in regard with granular models, granular computing is yet to be fully and exclusively explored, and its current structures and especially those related to fuzzy sets seem to be underdeveloped.

In 1997, Zadeh [8] introduced a strong relationship between granular data and fuzzy sets and developed the theory of fuzzy information granulation to provide a new angle of approach for tackling the problem of ambiguity and uncertainty. The theory of fuzzy information granulation (TFIG) is an informal method of using linguistic variables and fuzzy IF-THEN rules to make rational decisions in an environment full of uncertainty. Thus, this article is focused on this aspect of fuzzy theory and provides a method for defuzzification of fuzzy sets to achieve certainty in solution of real-world problems.

The concept of fuzzy sets (referring to the sets with imprecise and ambiguous nature) was first introduced in 1965 by Zadeh [9]. He expanded the notion of membership beyond the "zero-one" logic and utilized the dynamic infinite 
space between these values. In the mid-1980s, Japanese industrialists used this worthwhile concept to develop a scheme of fully automated subway controls, which demonstrated its real-world application encouraged a whole new wave of researchers to study its theoretical and practical potentials.

The foundation of conventional mathematics is based on real numbers and the process of defuzzifying and ranking fuzzy quantities-such as color or quality of goods-plays a significant role in data analysis, economics, and industrial systems, so an extensive amount of research has been dedicated to this specific subject.

Following the previous works [10-25], this study proposes a novel method for defuzzifying and ranking fuzzy numbers by using the mean value of Beta distribution. Some of the previous works in the literature are as follows.

In 1980, Yager [19] proposed a method for ranking fuzzy numbers based on their corresponding centroid-index. In 1981, the same author published another article proposing a method for ordering fuzzy subsets based on the integral of the mean of the level sets [20]. His method was capable of comparing crisp numbers, discrete fuzzy subsets, and continuous fuzzy subsets of the unit interval. In 1993, Choobineh and $\mathrm{Li}$ [13] introduced a fuzzy number ordering method, in which the membership function of fuzzy numbers did not need to be normal and convex. In addition, their index had some additional properties that made it more suitable for decision-making purposes. The concepts of expected interval and expected value were introduced in 1992 by Heilpern [21], who then used them to order fuzzy numbers. In 1998, Cheng [11] proposed a centroid-index ranking method for calculating the centroid-point of a fuzzy number. In 2000, Yao and $\mathrm{Wu}$ [17] used the decomposition principle and the crisp ranking system on $\mathrm{R}$ to construct a ranking system for fuzzy numbers. They used the decomposition principle to rewrite each fuzzy number as the union of all of its $\alpha$ cuts (where $\alpha \in(0,1)$ ) and then used the centroid-point of these $\alpha$-cuts to calculate the signed distance between the two fuzzy numbers. In 2001, Chen and Lu [18] developed an approximate approach for ranking fuzzy numbers based on the left and right dominance. To do so, they first defined the right and left limits of all $\alpha$-cuts for all fuzzy numbers and then defined the left and right dominance of a fuzzy number over another as the average difference of the left and right spreads. They then used an optimality index $B \in[0,1]$ to introduce the dominance of a fuzzy number over another as the convex combination of its left and right dominance. In 2003, S.-J. Chen and S.-M. Chen [16] introduced a procedure for ordering trapezoidal fuzzy numbers based on the center of gravity. The centroid-index ranking method presented in 2005 by Yong and Qi [12] employed TOPSIS to order the trapezoidal fuzzy numbers. In 2006, Asady and Zendehnam [10] presented a method for ranking fuzzy numbers by distance minimization. The fuzzy number ranking method proposed in 2008 by Chen and Wang [22] used $\alpha$-cuts for this purpose.

This paper aims to use statistical Beta distribution for defuzzifying and ranking fuzzy numbers, since it is the only distribution function that is bounded to $(0,1)$ and is zero outside this interval [26]. To do so, we can consider the surface inscribed within the projection of fuzzy number in the interval $(0,1)$ as a statistical population. In addition, parameters of this distribution could be set such that the resulting left spread, right spread, and mode fully match their corresponding values in the fuzzy number transformed to the interval $(0,1)$. We initiate the defuzzification process of a fuzzy number by obtaining the mean value of its corresponding Beta distribution. We then use simple arithmetic operations to determine the crisp number corresponding to that fuzzy number. We also provide a very simple algorithm for ordering fuzzy numbers based on their corresponding crisp real values and domains. The first stage of the algorithm is centripetal and considers the fuzzy number with greater corresponding real value as the greater fuzzy number, but when two fuzzy numbers have equal corresponding real values, it considers the one with righter intuitionistic position (no matter how slight) as the greater number. Our motivation for writing this paper is to provide an easy and tangible defuzzification method and a well-ordered algorithm for the ranking of fuzzy numbers. The method presented in this paper satisfies all reasonable properties defined by Wang and Kerre $[23,24]$ for ordering of triangular fuzzy numbers, but the mathematical complexity obstructed the process of proving the last property for trapezoidal fuzzy numbers.

The rest of this paper is organized as follows. Section 2 presents a brief introduction to fuzzy and statistical concepts and operations used in the paper. It also presents two theorems that constitute the basis of our method. Section 3 describes the proposed method of defuzzifying trapezoidal and triangular fuzzy numbers using the mean value of Beta distribution. This section also provides a fuzzy number ranking algorithm as well as ordinal properties to which this ranking method is applicable. In Section 4, several examples of fuzzy number ordering performed by the proposed method are presented. Section 5 uses 2 numerical examples to compare the proposed method of fuzzy number ranking with other methods developed for this purpose. The paper ends with Section 6, presenting conclusions.

\section{Preliminaries}

This section reviews some basic fuzzy and statistical concepts used in the rest of the paper. Two theorems that provide the basis of our method for obtaining crisp numbers corresponding to fuzzy numbers are introduced later in this section.

\subsection{Fuzzy Preliminaries}

Definition 1 (see [10]). A fuzzy number is a fuzzy set in the form of $\widetilde{a}: R \rightarrow[0,1]$ that satisfies the following conditions:

(1) $\tilde{a}$ is upper semicontinuous,

(2) $\tilde{a}(x)$ is zero outside the interval $[l, u]$,

(3) there exist real numbers $m_{1}, m_{2}$ such that $l \leq m_{1} \leq$ $m_{2} \leq u$ and

(3.1) $\tilde{a}(x)$ is increasing on $\left[l, m_{1}\right]$,

(3.2) $\tilde{a}(x)$ is decreasing on $\left[m_{2}, u\right]$,

(3.3) $\tilde{a}(x)=1, m_{1} \leq x \leq m_{2}$. 
If $m_{1}=m_{2}=m$ then the fuzzy number $\widetilde{a}=(l, m, u)$ is called the triangular fuzzy number and is defined as follows:

$$
\tilde{a}(x)= \begin{cases}\frac{x-l}{m-l} & l \leq x \leq m \\ \frac{u-x}{u-m} & m \leq x \leq u \\ 0 & \text { otherwise. }\end{cases}
$$

Moreover, the fuzzy number $\tilde{a}=\left(l, m_{1}, m_{2}, u\right)$ is called a trapezoidal fuzzy number and is defined as follows:

$$
\widetilde{a}(x)= \begin{cases}\frac{x-l}{m_{1}-l} & l \leq x \leq m_{1} \\ 1 & m_{1} \leq x \leq m_{2} \\ \frac{u-x}{u-m_{2}} & m_{2} \leq x \leq u \\ 0 & \text { otherwise. }\end{cases}
$$

The set of all fuzzy numbers is denoted by $E$. Furthermore, the set of all numbers that belong to the universal set $R$ such that $\widetilde{a}(x)>0$ is known as the supporter set of the fuzzy number $\widetilde{a}$.

2.2. Statistical Preliminaries. Let $S$ be the sample space of a random trial with a given probability value and let $X$ be the random variable defined as a real-valued function on $S$. If $X$ is a discrete random variable, the function $f(x)=P(X=x)$ for any specific value of $x$ within range of $X$ is called probability distribution. When $X$ is a continuous random variable, the function $f(x)$ is known as probability density function of $X$. Probability distributions and probability densities come in different types, including uniform density and Beta distribution just to name two.

The random variable $X$ is said to have Beta distribution if and only if its probability density is as follows:

$$
f(x)= \begin{cases}\frac{\Gamma(\alpha+\beta)}{\Gamma(\alpha) \cdot \Gamma(\beta)} x^{\alpha-1}(1-x)^{\beta-1} & 0<x<1 \\ 0 & \text { otherwise, }\end{cases}
$$

where $\alpha>0$ and $\beta>0$ are Beta distribution parameters. The mean of Beta distribution is obtained as follows:

$$
\mu=\frac{\alpha}{(\alpha+\beta)} .
$$

If $\alpha \geq 1$ and $\beta \geq 1$, then the curve of Beta function will be unimodal. When $\alpha>\beta$, the curve is said to have negative skewness and if $\alpha<\beta$, then the skewness is positive. For $\alpha=\beta$ the curve of Beta function is called symmetric. To better understand the described concept, see Figure 1, where $1<\alpha<\beta<2$. Given that the curve of Beta distribution is a unimodal one, $f^{\prime}(x)=0$ should have a unique solution. Solving the equation $f^{\prime}(x)=0$ gives the following relation between Beta distribution parameters:

$$
\beta=(\alpha-1)\left(\frac{1-x_{\mathrm{mod}}}{x_{\mathrm{mod}}}\right)+1,
$$

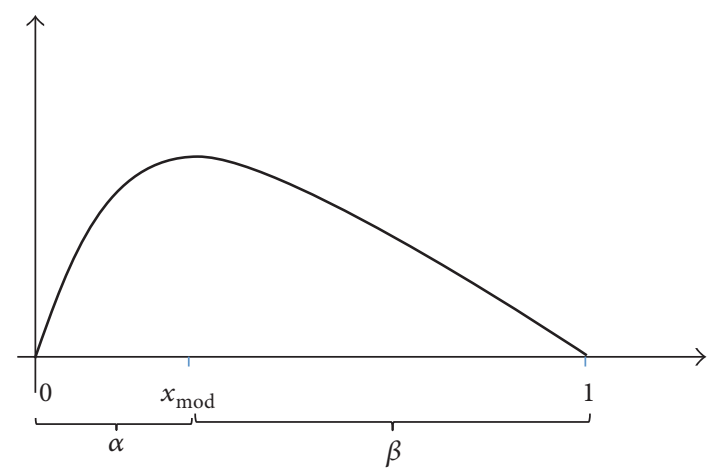

FIgURE 1: $\alpha<\beta$ and so the curve has positive skewness.

where $x_{\bmod } \in(0,1)$ is the point at which $f(x)$ has the maximum value. Hence given both values of $\alpha$ and $x_{\text {mod }}$, the $\beta$ parameter can be obtained from (5) and then the mean value of Beta distribution can be calculated by (4).

Uniform density is a special case of Beta distribution. The random variable $X$ is said to have uniform density if and only if its probability density is as follows:

$$
f(x)= \begin{cases}\frac{1}{q-p} & p<x<q \\ 0 & \text { otherwise. }\end{cases}
$$

The mean value of uniform density can be obtained by the following equation:

$$
\mu=\frac{p+q}{2} .
$$

Using the above-mentioned statistical preliminaries, a crisp number belonging to the interval $(0,1)$ and corresponding to the triangular fuzzy number can be obtained by (4) and (5) and a crisp number in the interval $(0,1)$ and corresponding to the trapezoidal fuzzy number can be obtained by (4), (5), and (7). The following theorems can then be used to calculate the real number corresponding to each fuzzy number in its domain.

Theorem 2. Let $a, b \in R$ and $<b$, and let $[a, b]$ be an interval on $R$. Then for every $\mu \in(a, b)$ there exists a unique number $\mu^{\prime} \in(a, b)$ and vice versa.

Proof. Suppose that $\mu \in(a, b)$ and let $\mu^{\prime}=(\mu-a) /(b-a)$; then we have $\mu^{\prime} \in(0,1)$.

The uniqueness of $\mu^{\prime}$ is proven by contradiction. Assuming that $\mu^{\prime} \in(0,1)$ and $\mu^{\prime \prime} \in(0,1)$ are two distinct numbers $\left(\mu^{\prime} \neq \mu^{\prime \prime}\right)$ corresponding to $\mu \in(a, b)$, the following relationships between $\mu$ and $\mu^{\prime}$ and between $\mu$ and $\mu^{\prime \prime}$ are established:

$$
\begin{aligned}
& \mu=\mu^{\prime}(b-a)+a, \\
& \mu=\mu^{\prime \prime}(b-a)+a .
\end{aligned}
$$

The above equations imply that $\mu^{\prime}=\mu^{\prime \prime}$ which contradicts the initial assumption; that is, $\mu^{\prime} \neq \mu^{\prime \prime}$. Thus, initial assumption is false and uniqueness of $\mu^{\prime}$ is proven. 
Theorem 3. For two distinct intervals $(a, b)$ and $(c, d)$ where $a-c \neq b-d$, assume that $\mu_{a} \in(a, b)$ and $\mu_{c} \in(a, b)$. If $\mu^{\prime}=\left(\mu_{a}-a\right) /(b-a)=\left(\mu_{c}-c\right) /(d-c)$, then $\mu_{a} \neq \mu_{c}$.

Proof. Let $(a, b)$ and $(c, d)$ be two distinct intervals. Then only one of the following is true:

(1) $a=c$ and $b \neq d$,

(2) $a \neq c$ and $b=d$,

(3) $a \neq c$ and $b \neq d$.

According to theorem's assumption, we have

$$
\frac{\left(\mu_{a}-a\right)}{(b-a)}=\frac{\left(\mu_{c}-c\right)}{(d-c)} .
$$

In the first case, we have $a=c$ and $b \neq d$. Assuming that $a=c$, it is supposed for contradiction that $\mu_{a}=\mu_{c}$. Relation (9) yields $b=d$ which contradicts the initial statement. Therefore the contradictory assumption is false, proving the claim. Statements (2) and (3) can be easily proved through similar arguments.

\section{Using Beta Distribution for Defuzzifying and Ranking Fuzzy Numbers}

In this section, for every fuzzy number $\widetilde{a}$, the mean value of its corresponding Beta distribution in its domain is considered as the crisp real number corresponding to $\tilde{a}$. Then the proposed method, which is based on crisp ranking system on $R$, is used to rank and order the fuzzy numbers.

3.1. Defuzzification of Triangular Fuzzy Numbers. Consider the triangular fuzzy number $\widetilde{a}=(l, m, u)$. To obtain the crisp real number corresponding to the triangular fuzzy number $\tilde{a}=(l, m, u)$, we first project $\tilde{a}$ on the interval $(0,1)$, which will be in the form of $\tilde{a}^{\prime}=((l-l) /(u-l),(m-l) /(u-l),(u-$ $l) /(u-l))=(0,(m-l) /(u-l), 1)$. Then we define the parameter $\alpha$ corresponding to the Beta distribution as follows:

$$
\alpha=\frac{m-l}{u-l}+1
$$

With the above definition, firstly, it is clear that $\alpha \geq 1$ and if $m \neq l$, then the Beta distribution curve will be unimodal. Secondly, if $-l=u-m$, which denotes a symmetric triangular fuzzy number, then $\alpha=\beta=3 / 2$ and the Beta distribution curve will be symmetric. Here, the left skewness of the Beta distribution curve is the left side spread of triangular fuzzy number divided by its support set.

In the Beta distribution corresponding to the projection of fuzzy number $\widetilde{a}=(l, m, u)$, we have $x_{\bmod }=(m-l) /(u-l)$ and by using (10) and substituting it into (5), we get

$$
\beta=\frac{u-m}{u-l}+1
$$

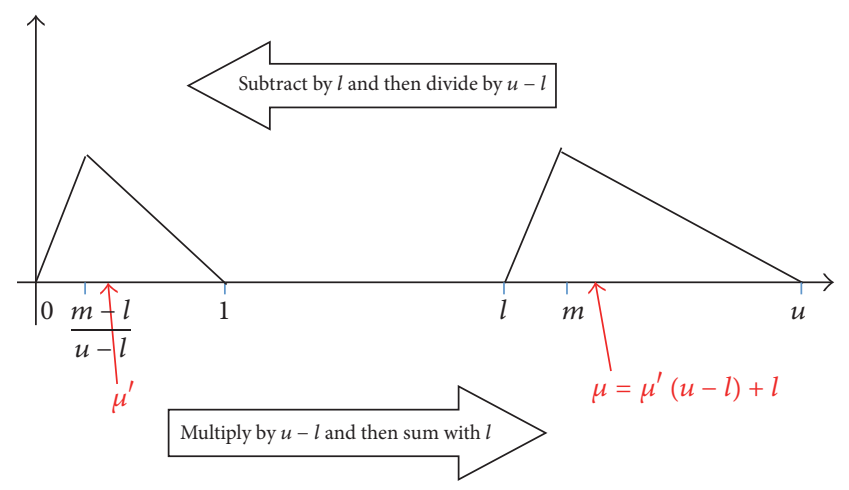

FIgURE 2: Transfer of the triangular fuzzy number to the interval $(0,1)$ and vice versa.

We use (4), (5), and (11) as shown below to calculate the mean value of Beta distribution corresponding to the fuzzy number:

$$
\begin{aligned}
\mu^{\prime} & =\frac{\alpha}{\alpha+\beta} \\
& =\frac{(m-l) /(u-l)+1}{(m-l) /(u-l)+1+(u-m) /(u-l)+1} \\
& =\frac{m+u-2 l}{3(u-l)} .
\end{aligned}
$$

The real number $\mu_{\tilde{a}}$, which is obtained (as shown below) by transferring $\mu^{\prime}$ from the interval $(0,1)$ to the interval $(l, u)$, is considered as the real number corresponding to the fuzzy number $\widetilde{a}=(l, m, u)$ :

$$
\mu_{\tilde{a}}=\mu^{\prime}(u-l)+l
$$

Figure 2 shows the manner of projecting the triangular fuzzy number on the interval $(0,1)$, and Figure 3 shows the manner of defining the Beta function corresponding to the projection of fuzzy number in the interval $(0,1)$.

Remark 4 . The crisp real number $\mu_{\tilde{a}}$ corresponding to the triangular fuzzy number $\widetilde{a}=(l, m, u)$ is obtained from the following relation:

$$
\mu_{\tilde{a}}=\frac{l+m+u}{3}
$$

3.2. Defuzzification of Trapezoidal Fuzzy Number. Consider the trapezoidal fuzzy number $\tilde{a}=\left(l, m_{1}, m_{2}, u\right)$. We use a combination of Beta distribution and uniform distribution to defuzzify this trapezoidal fuzzy number. To start, the interval $(l, u)$ is partitioned as shown below:

$$
(l, u)=A_{1} \cup A_{2} \cup A_{3},
$$

where $A_{1}=\left(l, m_{1}\right), A_{2}=\left[m_{1}, m_{2}\right]$, and $A_{3}=\left(m_{2}, u\right)$. We define the triangular fuzzy numbers corresponding to $A_{1}$ and $A_{3}$ as $\widetilde{a}_{1}=\left(l, m_{1}, m_{1}\right)$ and $\widetilde{a}_{3}=\left(m_{2}, m_{2}, u\right)$ and then obtain 


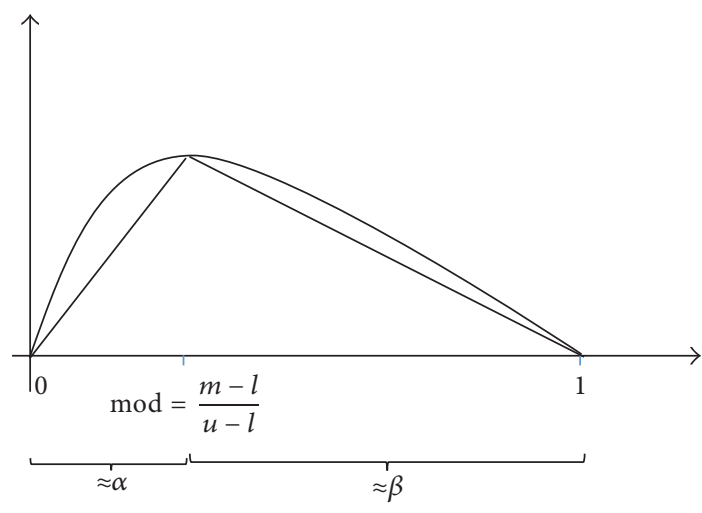

Figure 3: Graph of Beta function corresponding to the triangular fuzzy number.

their corresponding real numbers denoted by $a_{1}$ and $a_{3}$ as follows:

$$
\begin{aligned}
& a_{1}=\frac{l+m_{1}+m_{1}}{3}, \\
& a_{3}=\frac{m_{2}+m_{2}+u}{3} .
\end{aligned}
$$

In order to obtain the real number $a_{2}$ corresponding to the interval $A_{2}=\left[m_{1}, m_{2}\right]$, we use the uniform distribution and calculate its mean value via relation (7). The mean of the real numbers $a_{1}, a_{2}$, and $a_{3}$ is considered as the crisp real number corresponding to the trapezoidal fuzzy number.

Remark 5. The crisp real number $\mu_{\tilde{a}}$ corresponding to the trapezoidal fuzzy number $\widetilde{a}=\left(l, m_{1}, m_{2}, u\right)$ is obtained by the following relation:

$$
\mu_{\tilde{a}}=\frac{2 l+7 m_{1}+7 m_{2}+2 u}{18} .
$$

3.3. The Fuzzy Number Ranking Algorithm. Consider the fuzzy numbers $\widetilde{a}=\left(l_{\tilde{a}}, m_{\tilde{a}}, u_{\tilde{a}}\right)$ and $\widetilde{b}=\left(l_{\tilde{b}}, m_{\tilde{b}}, u_{\tilde{b}}\right)$. We take the following steps to order these numbers.

Step 1. Calculate the crisp real numbers $\mu_{\tilde{a}}$ and $\mu_{\tilde{b}}$ corresponding to $\tilde{a}$ and $\tilde{b}$.

Step 2.
(A) If $\mu_{\tilde{a}}>\mu_{\tilde{b}}$, then $\tilde{a}>\widetilde{b}$.
(B) If $\mu_{\tilde{a}}=\mu_{\tilde{b}}$ and $u_{\tilde{a}}>u_{\tilde{b}}$, then $\tilde{a}>\widetilde{b}$.
(C) If $\mu_{\tilde{a}}=\mu_{\tilde{b}}, u_{\tilde{a}}=u_{\tilde{b}}$, and $m_{\tilde{a}}>m_{\tilde{b}}$, then $\tilde{a}>\tilde{b}$.
(D) If $\mu_{\tilde{a}}=\mu_{\tilde{b}}, u_{\tilde{a}}=u_{\tilde{b}}$, and $m_{\tilde{a}}=m_{\tilde{b}}$, then $\tilde{a} \approx \tilde{b}$.

Note that, for trapezoidal fuzzy number, we consider $m=$ $m_{2}$ and use the symbols $>, \prec$, and $\approx$ to denote "greater than," "less than," and "equals" relations between fuzzy numbers.

We consider the following reasonable properties for the ordering approach; see $[23,24]$.

$\left(A_{1}\right)$ For an arbitrary finite subset $S$ of $E$ and $\tilde{a} \in S, \tilde{a} \succsim \tilde{a}$.
$\left(A_{2}\right)$ For an arbitrary finite subset $S$ of $E$ and $(\widetilde{a}, \widetilde{b}) \in S^{2}$, $\widetilde{a} \succsim \widetilde{b}$, and $\widetilde{b} \succsim \widetilde{a}$, we should have $\widetilde{a} \approx \widetilde{a}$.

$\left(A_{3}\right)$ For an arbitrary finite subset $S$ of $E$ and $(\widetilde{a}, \widetilde{b}, \widetilde{c}) \in S^{3}$, $\widetilde{a} \succsim \widetilde{b}$, and $\widetilde{b} \succsim \widetilde{c}$, we should have $\widetilde{a} \succsim \widetilde{c}$.

$\left(A_{4}\right)$ For an arbitrary finite subset $S$ of $E$ and $(\widetilde{a}, \widetilde{b}) \in S^{2}$, $\inf \operatorname{supp}(\widetilde{a})>\sup \operatorname{supp}(\widetilde{b})$, we should have $\tilde{a} \succsim \widetilde{b}$.

$\left(A_{4}^{\prime}\right)$ For an arbitrary finite subset $S$ of $E$ and $(\widetilde{a}, \widetilde{b}) \in S^{2}$, $\inf \operatorname{supp}(\widetilde{a})>\sup \operatorname{supp}(\widetilde{b})$, we should have $\tilde{a}>\widetilde{b}$.

$\left(A_{5}\right)$ Let $S$ and $S^{\prime}$ be two arbitrary finite subsets of $E$ in which $\tilde{a}$ and $\tilde{b}$ are in $S \cap S^{\prime}$. We obtain the ranking order $\tilde{a}>\widetilde{b}$ by Beta distribution method on $S^{\prime}$ if and only if $\widetilde{a}>\widetilde{b}$ by Beta distribution method on $S$.

$\left(A_{6}\right)$ Let $\tilde{a}, \tilde{b}, \widetilde{a}+\widetilde{c}$, and $\widetilde{b}+\widetilde{c}$ be elements of $E$. If $\widetilde{a} \succsim \widetilde{b}$, then $\tilde{a}+\tilde{c} \succsim \widetilde{b}+\tilde{c}$.

$\left(A_{6}^{\prime}\right)$ Let $\tilde{a}, \widetilde{b}, \widetilde{a}+\widetilde{c}$, and $\widetilde{b}+\widetilde{c}$ be elements of $E$ and $\widetilde{c} \neq \emptyset$. If $\tilde{a}>\widetilde{b}$, then $\tilde{a}+\widetilde{c}>\widetilde{b}+\widetilde{c}$.

$\left(A_{7}\right)$ Let $\tilde{a}, \tilde{b}, \tilde{a} \cdot \widetilde{c}$, and $\tilde{b} \cdot \widetilde{c}$ be elements of $E$ and $\widetilde{c} \geq 0$. $\tilde{a} \succsim \tilde{b}$ by Beta distribution method on $\{\tilde{a}, \tilde{b}\}$ implies $\widetilde{a} \cdot \widetilde{c} \succsim \widetilde{b} \cdot \widetilde{c}$ by one on $\{\widetilde{a} \cdot \widetilde{c}, \widetilde{b} \cdot \widetilde{c}\}$.

While we could only prove the properties $A_{1}$ to $A_{6}^{\prime}$ for the trapezoidal fuzzy numbers, all of these properties are satisfied for the triangular fuzzy numbers.

In the next section, we use the proposed method to order several fuzzy numbers.

\section{Numerical Examples}

In this section, we first use the mean value of Beta distribution to obtain the crisp real numbers corresponding to three sets of fuzzy numbers, and then use the proposed algorithm to order these numbers.

Set 1. Consider three fuzzy numbers $\widetilde{a}, \widetilde{b}$, and $\widetilde{c}$ as follows:

$$
\begin{aligned}
& \tilde{a}=(1,3,5), \\
& \widetilde{b}=(3,5,8), \\
& \widetilde{c}=(3,8,12) .
\end{aligned}
$$

The Beta distribution method yields their corresponding real numbers as follows:

$$
\begin{aligned}
& \mu_{\tilde{a}}=3, \\
& \mu_{\tilde{b}}=5.333, \\
& \mu_{\tilde{c}}=7.667 .
\end{aligned}
$$

The proposed algorithm returns $\widetilde{a} \prec \widetilde{b} \prec \widetilde{c}$ (see Figure 4).

Set 2. Consider the fuzzy numbers $\tilde{a}=(2,3,4)$ and $\tilde{b}=$ $(0,3,6)$. According to the proposed method, the crisp real numbers corresponding to $\tilde{a}$ and $\tilde{b}$ are equal $\left(\mu_{\tilde{a}}=\mu_{\tilde{b}}=\right.$ 3 ), but as Figure 5 shows, based on the hatched area, the proposed algorithm returns $\tilde{a}<\tilde{b}$. 

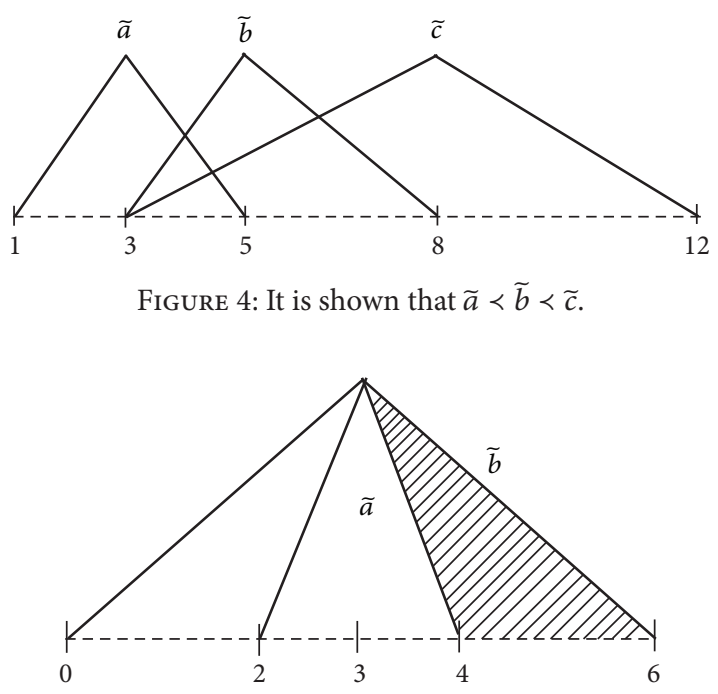

FIgURE 5: It is shown that $\tilde{a} \prec \tilde{b}$.

Set 3. Consider the following four fuzzy numbers $\tilde{a}, \widetilde{b}, \widetilde{c}$, and $\widetilde{d}$ and their corresponding crisp real numbers obtained from Beta distribution method:

$$
\begin{aligned}
& \tilde{a}=(4,6,10,12) \longrightarrow \mu_{\tilde{a}}=8, \\
& \widetilde{b}=(4,7,9,12) \longrightarrow \mu_{\tilde{b}}=8, \\
& \tilde{c}=(8,10,12,14) \longrightarrow \mu_{\tilde{c}}=9.778, \\
& \tilde{d}=(6,8,10) \longrightarrow \mu_{\tilde{d}}=8 .
\end{aligned}
$$

It is clear that $\tilde{c}$ is greater than other fuzzy numbers, because $\mu_{\tilde{c}}$ is greater than other obtained crisp real numbers. Also $\mu_{\tilde{a}}=$ $\mu_{\tilde{b}}=\mu_{\tilde{d}}=8$. Meanwhile $u_{\tilde{d}}$ is less than $u_{\tilde{a}}$ and $u_{\tilde{b}}$, so $\tilde{d}$ is the smallest fuzzy number in the set. Since $u_{\tilde{a}}=u_{\tilde{b}}$, to determine their ordering we need to compare their $m_{2}$ values. Hence we have $\widetilde{b}<\tilde{a}$.

The proposed method determines the ordering of the above fuzzy numbers as $\widetilde{d}<\widetilde{b}<\widetilde{a}<\widetilde{c}$ (see Figures 6 and 7). As Figure 6 shows, although $\mu_{\tilde{a}}=\mu_{\tilde{b}}$, based on the hatched area, the proposed algorithm announces that $\widetilde{b}<\tilde{a}$.

\section{The Comparison of Beta Distribution Method with Other Existing Methods}

In this section we compare our method of ranking fuzzy numbers with other methods developed for this purpose. We do so by using two sets of fuzzy numbers.

Set 1. Consider the set of fuzzy numbers containing $\widetilde{a}=$ $(0.1,0.3,0.5), \widetilde{b}=(0.2,0.3,0.4)$, and $\widetilde{c}=(1,1,1)$. Table 1 shows the ranking of these three numbers according to the methods of Chen and Wang [22], Yong and Qi [12], and S.-J. Chen and S.-M. Chen [16], in addition to the results obtained by the proposed method. As can be seen, the proposed

\begin{tabular}{|c|c|c|c|c|}
\hline \multirow{2}{*}{ Ranking method } & \multicolumn{3}{|c|}{ Set of fuzzy numbers } & \multirow{2}{*}{ Ranking results } \\
\hline & $\tilde{a}$ & $\widetilde{b}$ & $\widetilde{c}$ & \\
\hline Chen and Wang & 0.0992 & 0.1021 & 0.4737 & $\tilde{a} \prec \tilde{b} \prec \tilde{c}$ \\
\hline Yong and Qi & 0.6214 & 0.6244 & 1 & $\tilde{a} \prec \tilde{b} \prec \tilde{c}$ \\
\hline $\begin{array}{l}\text { S.-J. Chen and } \\
\text { S.-M. Chen }\end{array}$ & 1.2359 & 1.2674 & 2 & $\tilde{a} \prec \tilde{b} \prec \tilde{c}$ \\
\hline $\begin{array}{l}\text { Proposed } \\
\text { method }\end{array}$ & 0.3 & 0.3 & 1 & $\tilde{b} \prec \widetilde{a} \prec \tilde{c}$ \\
\hline
\end{tabular}
method returns equal crisp real numbers for $\widetilde{a}$ and $\widetilde{b}$, but since $u_{\tilde{a}}>u_{\tilde{b}}$, we conclude that $\tilde{a}>\widetilde{b}$. The proposed algorithm,
TABLE 1: Ranking results for the first set of fuzzy.

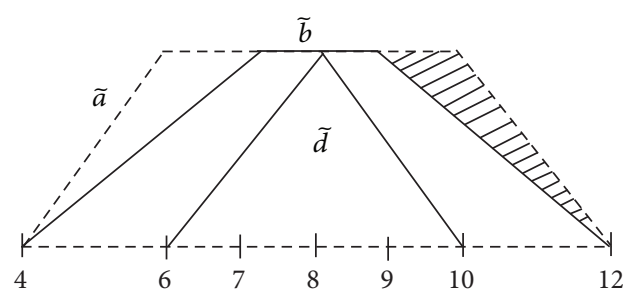

FIgURe 6: It is shown that $\tilde{d} \prec \widetilde{b} \prec \tilde{a}$.

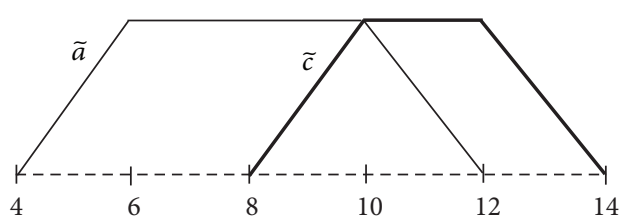

FIgURE 7: It is shown that $\widetilde{a} \prec \widetilde{c}$.

which is based on the mean value of Beta distribution, ranks these three fuzzy numbers as $\widetilde{b}<\widetilde{a}<\widetilde{c}$ contradicting the result of other ranking methods used for comparison (see Figure 8).

Set 2. Consider the following three fuzzy numbers $\widetilde{a}, \widetilde{b}$, and $\widetilde{c}:$

$$
\begin{aligned}
& \tilde{a}=(0,0.4,0.7,0.8), \\
& \widetilde{b}=(0.2,0.5,0.9), \\
& \widetilde{c}=(0.1,0.6,0.8) .
\end{aligned}
$$

The ranking of these three fuzzy numbers obtained by the methods proposed in $[10,13,15,17,20,23,25]$ and the result of Beta distribution method are shown in Table 2.

The method proposed in this paper calculates the real numbers corresponding to fuzzy numbers $\widetilde{a}, \widetilde{b}$, and $\widetilde{c}$ as $0.516,0.533$, and 0.5 and therefore determines the order of these fuzzy numbers as $\widetilde{c}<\widetilde{a}<\widetilde{b}$, which is only consistent with the distance method proposed by Wang, thus contradicting the results obtained via other methods. For a better understanding, see Figures 9 and 10.

\section{Conclusion}

In this paper, we proposed a simple method for obtaining the crisp real number corresponding to a fuzzy number by using 
TABLE 2: Ranking results for the second set of fuzzy.

\begin{tabular}{|c|c|c|c|c|}
\hline \multirow{2}{*}{ Authors } & \multicolumn{3}{|c|}{ Set of fuzzy numbers } & \multirow{2}{*}{ Ranking results } \\
\hline & $\tilde{a}$ & $\tilde{b}$ & $\tilde{c}$ & \\
\hline Choobineh and Li & 0.5 & 0.5833 & 0.6111 & $\tilde{a} \prec \widetilde{b} \prec \widetilde{c}$ \\
\hline Baldwin and Guild & 0.4 & 0.42 & 0.42 & $\tilde{a} \prec \tilde{b} \approx \tilde{c}$ \\
\hline Yager & 0.45 & 0.525 & 0.55 & $\tilde{a} \prec \tilde{b} \prec \tilde{c}$ \\
\hline Yao and $\mathrm{Wu}$ & 0.475 & 0.525 & 0.525 & $\tilde{a} \prec \tilde{b} \approx \tilde{c}$ \\
\hline Wang centroid method & 0.1967 & 0.1778 & 0.1667 & $\tilde{c} \prec \tilde{b} \prec \tilde{a}$ \\
\hline Wang distance & 0.6284 & 0.6289 & 0.6009 & $\tilde{c} \prec \tilde{a} \prec \widetilde{b}$ \\
\hline Asady distance & 0.475 & 0.525 & 0.525 & $\tilde{a} \prec \tilde{b} \approx \tilde{c}$ \\
\hline Proposed method & 0.516 & 0.533 & 0.5 & $\tilde{c} \prec \tilde{a} \prec \tilde{b}$ \\
\hline
\end{tabular}

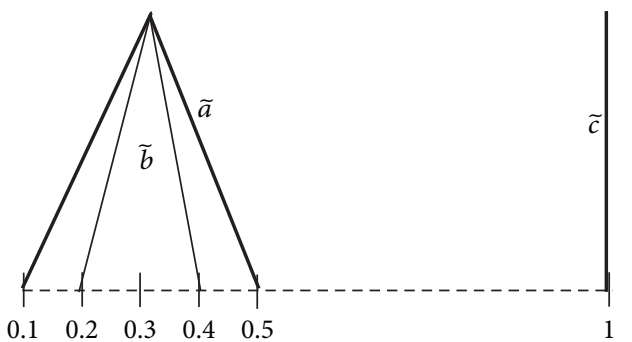

FIGURE 8: It is shown that $\widetilde{b} \prec \widetilde{a} \prec \widetilde{c}$.

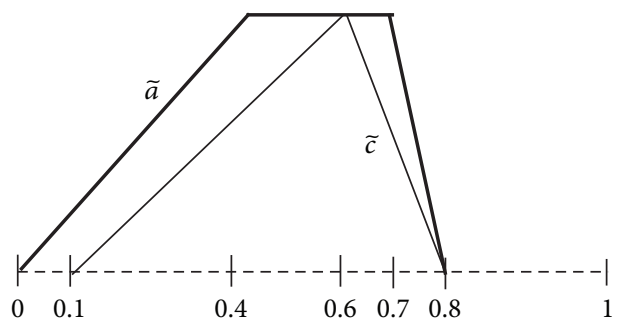

FIGURE 9: It is shown that $\widetilde{c} \prec \tilde{a}$.

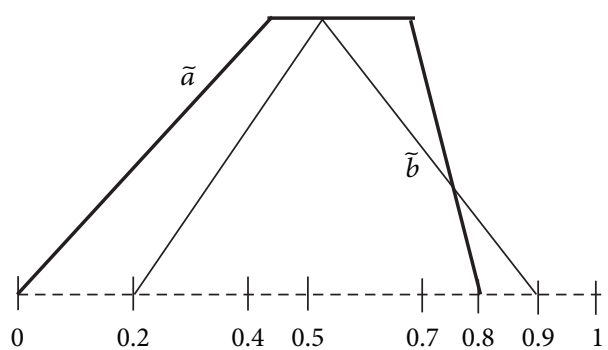

Figure 10: It is shown that $\tilde{a}<\tilde{b}$.

the mean value of Beta distribution and showed that this crisp real number could be obtained via simple mathematical operations. We also introduced a novel algorithm for ranking and ordering fuzzy numbers and reviewed the reasonable properties established for this algorithm and ultimately showed that the proposed method is a complete methodology for ranking fuzzy numbers. In the end, we used numerical examples to demonstrate the performance of our method and then compared it with other methods developed with similar objectives.

\section{Competing Interests}

The authors declare that there is no conflict of interests regarding the publication of this paper.

\section{References}

[1] W. Pedrycz and S. M. Chen, Granular Computing and DecisionMaking: Interactive and Iterative Approaches, Springer, Heidelberg, Germany, 2015.

[2] Y. Y. Yao, "Granular computing," Computer Science, vol. 31, pp. $1-5,2004$.

[3] J. M. Mendel, "A comparison of three approaches for estimating (synthesizing) an interval type-2 fuzzy set model of a linguistic term for computing with words," Granular Computing, vol. 1, no. 1, pp. 59-69, 2016.

[4] A. Skowron, A. Jankowski, and S. Dutta, "Interactive granular computing," Granular Computing, vol. 1, no. 2, pp. 95-113, 2016.

[5] G. Wilke and E. Portmann, "Granular computing as a basis of human-data interaction: a cognitive cities use case," Granular Computing, vol. 1, no. 3, pp. 181-197, 2016.

[6] L. Livi and A. Sadeghian, "Granular computing, computational intelligence, and the analysis of non-geometric input spaces," Granular Computing, vol. 1, no. 1, pp. 13-20, 2016.

[7] M. Antonelli, P. Ducange, B. Lazzerini, and F. Marcelloni, "Multi-objective evolutionary design of granular rule-based classifiers," Granular Computing, vol. 1, pp. 37-58, 2016.

[8] L. A. Zadeh, "Toward a theory of fuzzy information granulation and its centrality in human reasoning and fuzzy logic," Fuzzy Sets and Systems, vol. 90, no. 2, pp. 111-127, 1997.

[9] L. A. Zadeh, "Fuzzy sets," Information and Computation, vol. 8, pp. 338-353, 1965.

[10] B. Asady and A. Zendehnam, "Ranking fuzzy numbers by distance minimization," Applied Mathematical Modelling, vol. 31, no. 11, pp. 2589-2598, 2007.

[11] C.-H. Cheng, "A new approach for ranking fuzzy numbers by distance method," Fuzzy Sets and Systems, vol. 95, no. 3, pp. 307317, 1998 .

[12] D. Yong and L. Qi, "A TOPSIS-based centroid-index ranking method of fuzzy numbers and its application in decisionmaking," Cybernetics and Systems, vol. 36, no. 6, pp. 581-595, 2005.

[13] F. Choobineh and H. Li, "An index for ordering fuzzy numbers," Fuzzy Sets and Systems, vol. 54, no. 3, pp. 287-294, 1993.

[14] H. J. Zimmermann, Fuzzy Set Theory and Its Applications, Kluwer-Nijhoff, Boston, Mass, USA, 2nd edition, 1991.

[15] J. F. Baldwin and N. C. Guild, "Comparison of fuzzy sets on the same decision space," Fuzzy Sets and Systems, vol. 2, no. 3, pp. 213-231, 1979.

[16] S.-J. Chen and S.-M. Chen, "Fuzzy risk analysis based on similarity measures of generalized fuzzy numbers," IEEE Transactions on Fuzzy Systems, vol. 11, no. 1, pp. 45-56, 2003.

[17] J.-S. Yao and K. Wu, "Ranking fuzzy numbers based on decomposition principle and signed distance," Fuzzy Sets and Systems, vol. 116, no. 2, pp. 275-288, 2000.

[18] L.-H. Chen and H.-W. Lu, "An approximate approach for ranking fuzzy numbers based on left and right dominance," Computers and Mathematics with Applications, vol. 41, no. 12, pp. 1589-1602, 2001.

[19] R. R. Yager, “On a general class of fuzzy connectives," Fuzzy Sets and Systems, vol. 4, no. 3, pp. 235-242, 1980. 
[20] R. R. Yager, "A procedure for ordering fuzzy subsets of the unit interval," Information Sciences, vol. 24, no. 2, pp. 143-161, 1981.

[21] S. Heilpern, "The expected value of a fuzzy number," Fuzzy Sets and Systems, vol. 47, no. 1, pp. 81-86, 1992.

[22] S.-M. Chen and C.-H. Wang, "Fuzzy risk analysis based on ranking fuzzy numbers using $\alpha$-cuts, belief features and signal/noise ratios," Expert Systems with Applications, vol. 36, no. 3, pp. 5576-5581, 2009.

[23] X. Wang and E. E. Kerre, "Reasonable properties for the ordering of fuzzy quantities. (I)," Fuzzy Sets and Systems, vol. 118, no. 3, pp. 375-385, 2001.

[24] X. Wang and E. E. Kerre, "Reasonable properties for the ordering of fuzzy quantities (II)," Fuzzy Sets and Systems, vol. 118, no. 3, pp. 387-405, 2001.

[25] Y.-M. Wang, J.-B. Yang, D.-L. Xu, and K.-S. Chin, "On the centroids of fuzzy numbers," Fuzzy Sets and Systems, vol. 157, no. 7, pp. 919-926, 2006.

[26] J. E. Freund, Mathematical Statistics, Prentice-Hall, 5th edition, 1992. 

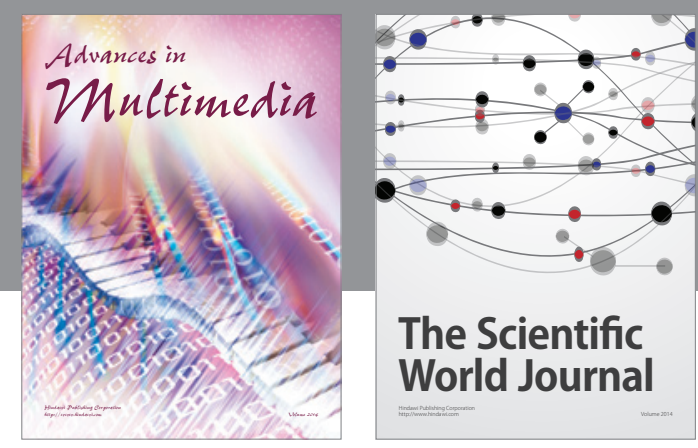

The Scientific World Journal
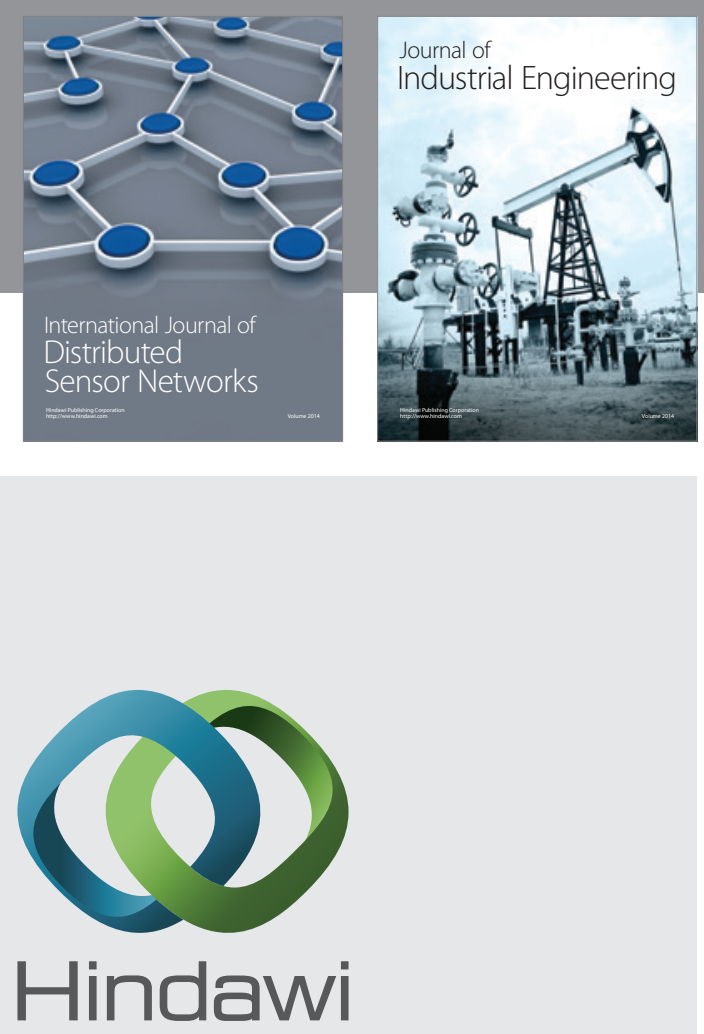

Submit your manuscripts at

http://www.hindawi.com

\section{Computer Networks} and Communications
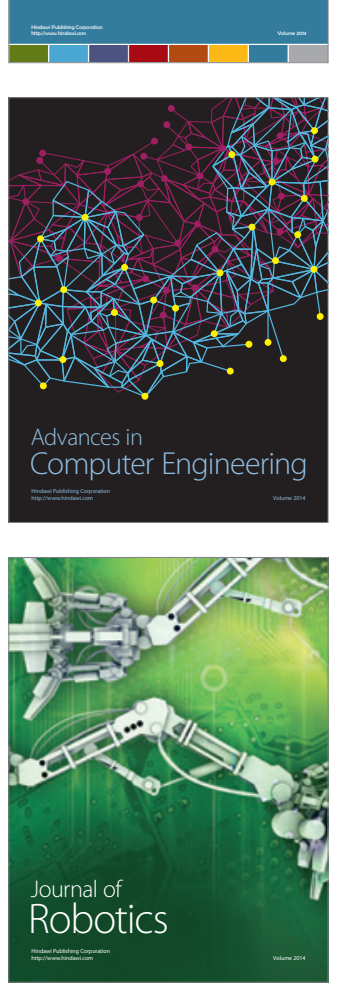
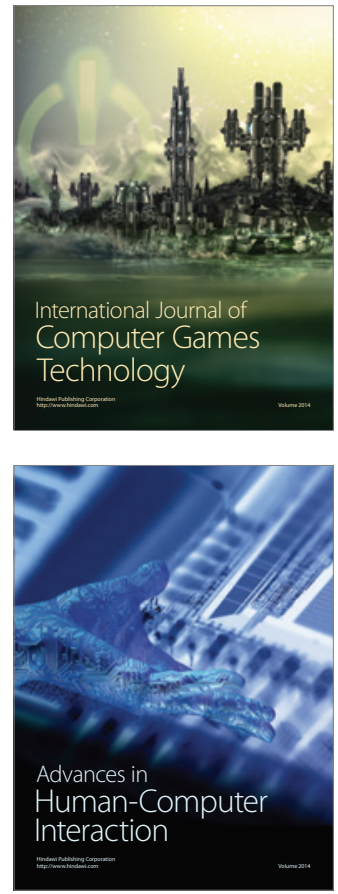
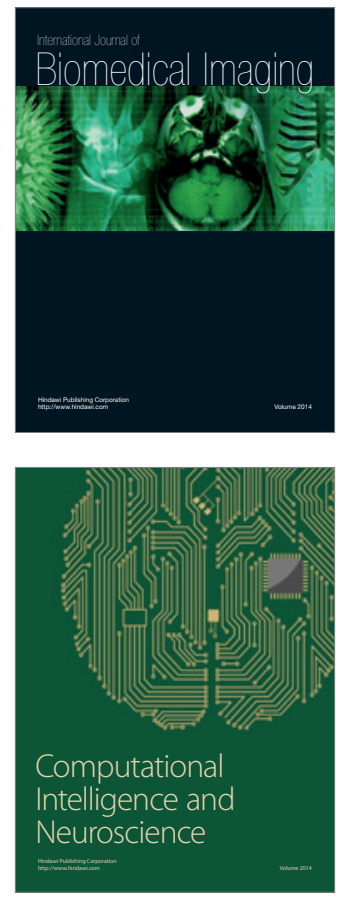
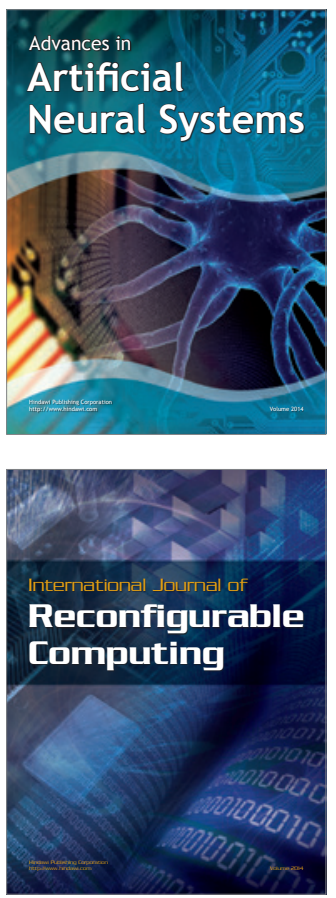
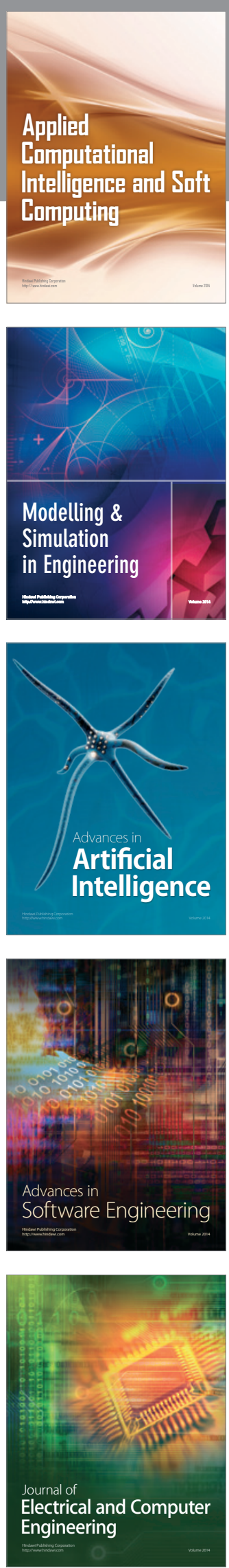